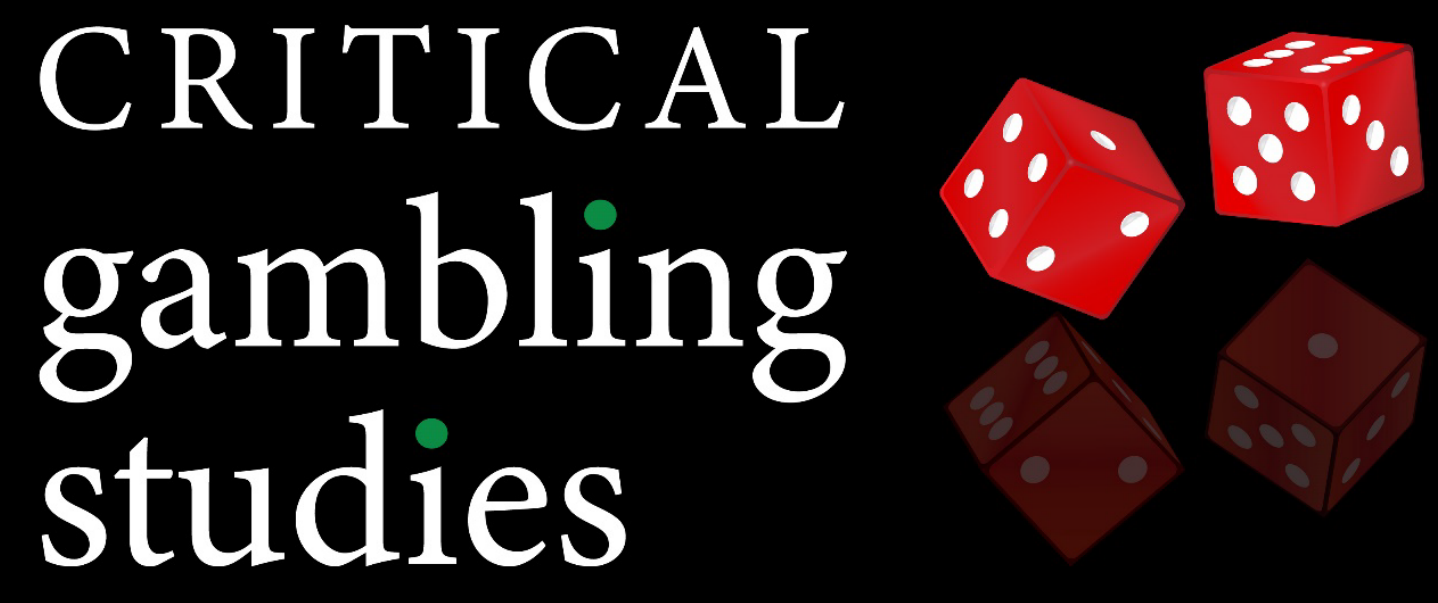

ISSN: 2563-190X. Available Open Access at https://criticalgamblingstudies.com

\title{
Editorial: What are Critical Gambling Studies?
}

Fiona Nicoll, Kate Bedford, Angela Rintoul, Charles Livingstone, Emma Casey

APA Citation: Nicoll, F., Bedford, K., Rintoul, A., Livingstone, C., \& Casey, E. (2022). Editorial: What are Critical Gambling Studies? Critical Gambling Studies, 3(1), i-v. https://doi.org/10.29173/cgs135 


\title{
EDITORIAL
}

\section{Editorial: What Are Critical Gambling Studies?}

\author{
Fiona Nicoll, Kate Bedford, Angela Rintoul, Charles Livingstone, Emma Casey
}

Available Open Access from https://doi.org/10.29173/cgs135

\section{The Emergence of CGS}

Critical Gambling Studies was initiated by a group of scholars between 2015 and 2017. We work in various disciplines, including public health, law, cultural studies, sociology, political science, and anthropology. Together we identified a significant gap in academic publishing for high quality, internationally significant research on gambling that did not conform to existing dominant theoretical frameworks, methodologies, and funding arrangements.

The need for such a journal was increasingly apparent. One of us had recently attended an academic conference held at a major casino in the US, where a keynote address was given by the casino's owner. (He would later resign, after reports of numerous allegations of harassment and assault against employees). Another was trying to raise awareness of the limits of male-dominated, Eurocentric approaches to gambling. Humanities and social science academics with careers built on gambling research found it almost impossible to recruit graduate students due to the perception that gambling knowledge was the domain of psychologists and addiction researchers, as well as an enduring stigma surrounding those who gamble. A related challenge for some of us was the unconscious bias that academic researchers can bring to the study of Indigenous and other marginalized populations. Public health researchers struggled to find support to expand a limited evidence base, combined with political pressure to maintain the status quo of gambling liberalization. Political economists and area studies experts found it difficult to convince mainstream scholars in the field of the importance of detailed casino studies and quantitative analyses of gambling's social benefits and costs. For others, an obvious deficit of critical thinking in mainstream gambling studies was the key motivating factor. Powerful academics working in the field mounted extraordinary responses to critiques of their perspective, further demonstrating the pressing need for a new journal.

Indeed, the project's formation was inextricable from concerns about a field dominated primarily by psychologists conducting research on 'problem' or 'pathological' gambling and (more recently) videogaming 'addiction.' ${ }^{\prime}$ Researchers taking a different approach, or whose research presented uncomfortable findings, were routinely excluded, silenced, intimidated or dismissed as ideologues and activists. These concerns animated our conversations about the need for a new journal to bring together scholars interested in changing this paradigm. In this regard there are at least three broad and related concerns shared by many scholars aligned with the project of critical gambling studies.

Firstly, we are troubled by the apparent consensus between governments, some treatment providers, the gambling industry, and some academics that people who experience harms from gambling are "problem gamblers" who need to be "cured" so they can "gamble responsibly", or directed to use flawed self-exclusion tools. We are concerned about the conflicts of interest that arise when the promotion of "responsible gambling" becomes the shared goal of governments, gambling operators, and academic researchers. A focus on individual gamblers' pathology is not only stigmatizing; it draws attention and funding away from other sites of responsibility, including that of designers and operators to provide less harmful products, and governments to effectively regulate how gambling is provided.

Secondly, we see the urgent need for truly interdisciplinary approaches, based on a genuine

'The term 'addiction' is especially contested in the videogaming arena. 
interest in mutual learning. We are skeptical about a thin veneer of interdisciplinarity that cloaks some journals and many scholarly meetings predominantly grounded within psy-sciences and STEM disciplines from which technocratic solutions to gambling harms are increasingly sought. Those of us who do work within these disciplines are attentive to the politics of disciplinarity in a context where research in the humanities is continually interrogated about its economic and epistemological value, and its methodological rigour. This is in stark contrast to a lack of similar scrutiny of some gambling research published in psy-science fields, even if methodologically weak and theoretically incoherent. This politics of academic disciplinarity highlights an urgent need for a range of new avenues to cultivate critical research on gambling such as conferences, symposia and blogs.

Thirdly, we are conscious that the rapid technological development of gambling products and their integration within everyday digital practices and platforms, and the expansion of marketing to new consumers, has significant harmful consequences. The transformations driven by networked, digital technologies, for example, have enabled gambling and gambling-like phenomena to diffuse into surprising spaces, presenting significant challenges for regulatory regimes that were developed in some cases before the widespread use of the internet. The rapid expansion of markets in low- and middle-income countries is of particular concern, with inadequate regulatory oversight making populations vulnerable to targeting by powerful corporations. We find many existing studies of these developments lack grounding in key literatures (e.g., in national and regional area studies; in socio-technical and science and technology studies; computer science; design; marketing; critical public health; Indigenous studies). More work is needed to understand interlocking factors that make the production and dissemination of rigorous, interdisciplinary, and wide-ranging research about radical transformations of platforms for gambling, investment, and play particularly challenging. While such research does exist, it is rarely published in leading gambling journals. In fact, it is rarely even engaged by them.

Our goal is to support research that is driven by genuine intellectual inquiry: independent from commercial and charitable gambling sectors; from states/government actors; and from anti-gambling pressure groups and faith-based groups. Critical Gambling Studies not only creates space for critiques of dominant policy solutions; it demands that alternatives are considered. Sometimes alternatives gain traction, as is happening with the public health approach to gambling harm in NZ and the UK, and evident in serious editorial attention by prominent medical publications including The Lancet and the British Medical Journal. Relatedly, there is now increasing attention being given to improving transparency in the funding of mainstream gambling research: in the UK, for example, in February 2022 the National Health Service announced it was severing its links with a leading gambling charity due to concerns over its connections to the gambling industry. While these shifts are significant achievements, we believe that critical gambling studies should not be restricted to 'critical law/policy studies of gambling' or 'a critique of problem gambling from a broader public health perspective'. While gambling harms (including mental illness, disability, and suicide) will likely remain core topics of concern, Critical Gambling Studies must be more than an instrument for determining or implementing better gambling law/policy/treatment/prevention.

We welcome work seeking to inform improvements to legislation, regulation, policy, treatment, and public health and see the urgent need for this in reducing harms related to gambling. However, we also welcome work driven by other objectives, including the pure research goal of achieving a better understanding of gambling as a widespread cultural practice in many societies. For example, there is a need to understand popular forms of gambling in everyday life (such as bingo and raffles) which receive much less attention than new and/or glamorized forms (such as eSports and poker) and relatively stigmatized forms (such as EGMs).

Across the platform of the journal as a whole, we are committed to understanding impacts of gambling beyond the limited focus on the individual, and to making space for perspectives that have been excluded from other gambling studies publications. For example, we seek to generate conversations about the connections between the local, national/state, and global dimensions of gambling practice and governance/regulation. Accordingly, we welcome comparative research on gambling practices and harmminimisation that teases out the links and distinctions between gambling and compulsive consumptions such as alcohol, cannabis, and tobacco.

We embrace mixed methods, and quantitative and qualitative studies, but we are attentive to the limits of methods in all cases: we encourage authors to discuss these limits in their submissions. We also reject the conflation of quantitative research with a monolithic "scientific method", understanding science instead as a field in which methods, theoretical frameworks, and hypotheses exist in states of contestation and flux. Rather than seeking to transplant existing theoretical frameworks into the gambling studies arena, we understand critical gambling studies as an epistemological intervention. We embrace the provisional and context-specific knowledge that emerges when distinct and overlapping disciplines begin to converge on new research problems, and defend its value even when states, policymakers, and industry are unable to discern immediate relevance or utility.

To broaden the knowledge base and accessibility of our field, we engage the expertise and lived experiences 
of non-academic professionals who can offer more detailed perspectives on specific topics. To this end we also engage our editorial board members in blogs on current developments in the rapidly shifting landscape of gambling production, regulation and consumption. Bringing those working at the coalface of gambling and gaming design and regulation into conversation with academic researchers enriches the knowledge base of a field which is otherwise dominated by investigations of pathology.

Our first three issues of Critical Gambling Studies and related blog series demonstrate that our field has independent academic merit. By creating a new arena for publication, we have been able to showcase new and original thinking about gambling across academic disciplines including history; political science, surveillance studies, critical theory, sociology, social theory and, importantly, decolonial and Indigenous studies. Special issues have featured early career researchers working across academic disciplines as well as showcased critical thinking within the field of Indigenous gambling studies.

\section{What does it mean to Promote 'Critical Approaches' to Gambling Research?}

Describing our approach to gambling as "critical" is not to promote or strictly adhere to existing models of critical theory, including but not restricted to frameworks provided by Marxist, feminist, critical race, critical disability, queer and decolonial scholars. Rather, the term "critical" designates a departure from scholarship that seeks to detach itself from the sphere of formal politics on one hand, and social justice or activist movements on the other. An important contribution of the Critical Gambling Studies project is its methodological diversity, and its broad interest in how gambling relates to power and politics; that breadth cannot, in all cases, be reduced to an attempt to influence policy. In an early intervention into socio-legal studies, Austin Sarat and Susan Silbey (1988) explored the way that intellectual enquiry was shaped, and sometimes limited, by "the pull of the policy audience". For them, that pull involved narrowing a concern with politics to an attempt to influence policy. This can be very seductive for marginalised academic subfields lacking power and resources, and needing sponsorship from external actors for legitimacy. To be critical in this context is to move beyond the "pull of the policy audience" and expand the lens of what counts as political by accepting our responsibilities, not only as researchers employed by universities, but also as highly educated professionals and citizens within democratic societies.

Commercial gambling exemplifies the messy business of governing complex capitalist societies, and it is necessary for scholarship to attend to this. A critical approach complements the existing focus on gambling harms by exploring gambling as a system, where structural relations of power shape and limit the capacities of individuals and institutions. Critical Gambling Studies welcomes research that addresses issues arising from the unprecedented pace of technological transformations: given the lived effects of such "innovation", we must identify the stakes at play when technological change drives accumulation and regulation. Addressing the unintended consequences of compliance and policing measures is also of far reaching (if understated) importance in gambling research. For example, digital surveillance measures using artificial intelligence and facial recognition incorporate bias, which can adversely affect racialised peoples who gamble in casinos. The expansion of cashless gambling platforms also creates personal data and consumer profiles over which players have extremely limited control.

These issues are not confined to the world of gambling, but are intimately entangled in the ways that academic publishing is produced and how bibliometrics are tied to the career progression of individual researchers and the institutional aspirations of universities that employ us. In a context where a handful of multinational companies publish the majority of journals for profit by placing academic research behind paywalls, Open Access models and publishing platforms have emerged to expand public access to knowledge. As an Open Access, double blind peer-reviewed journal, Critical Gambling Studies is part of a transnational movement of knowledge producers who value research quality over quantity and public access over private, for-profit control of research. Our digital open access model also tries to challenge environmentally unsustainable practices such as printonly journal production.

A further sense in which critical gambling studies are critical is through the embrace of a professional ethos that extends beyond avoiding conflicts of interests in funding and reviewing. We seek to reflect on, and improve, other ways that scholars interact in the process of evaluating, selecting, and editing manuscripts. While not all manuscripts are accepted for peer review in our journal, we will always provide constructive criticism for authors and recommendations to alternative publication outlets where possible. We guide reviewers to provide clear and constructive, developmental feedback with reference to clear and explicit criteria. When manuscripts are accepted, we particularly support early career researchers and non-academic authors through the process of responding to peer-review and/or copyediting feedback. Consulting with experts, we have drafted an Equity, Diversity and Inclusion statement and a copy-editing guide that is attentive to inclusive language, and we work closely with authors to honour their voices. The final version of this statement will be posted on our website after completion of editorial board review. As part of our commitment to intersectional frameworks of analysis we actively seek work from researchers and non-academic experts from 
communities that are most directly affected by gambling, even as they currently form a minority of researchers in the field. As members of the editorial board, we hold ourselves to implement the values within our EDI statement as a standard of professionalism in all our work. Our critical ethos reflects our continuing support for an emerging generation of gambling researchers who will inherit a field that is less compromised by powerful stakeholders, and more able to comprehensively understand gambling, including the ways it facilitates creativity and conviviality as well as extraction and pathology.

Critical approaches to gambling research are also, in a sense, aleatory. They must evolve as gambling evolves. Critical Gambling Studies also provides, therefore, a platform for experimentation, for continuing to work out what it means to practice and embody critique as it relates to changing gambling realities and the ways they are understood.

\section{The Current Issue of the Journal}

This special issue demonstrates how the scope of critical gambling studies continues to expand. We open the issue with a fascinating piece showing how the study of memoir aids a richer understanding of women's experiences in the highly masculinized spaces of competitive poker. Julie Rak analyzes the gender politics of memoirs by two prominent professional poker players (Annie Duke and Victoria Coren), arguing that these memoirs are a good place to look for "perifeminist" strategies. James Cosgrave's piece examines the instrumentalization of gambling for state and industry ends. Using the work of Max Weber and Jurgen Habermas to better understand the contemporary cultural significance of gambling in an era of mass liberalization, he argues that gambling instrumentalization is linked to the development (and regulation) of late modern subjectivity. While these opening pieces use humanist and sociological methods, Jani Selin's article demonstrates the importance and relevance of interpretive political analysis to gambling research. $\mathrm{He}$ analyzes parliamentary speech about gambling harm and revenue from Finnish politicians as a form of political action, and he offers a new approach to how politics might be approached in critical gambling studies.

We also offer a number of pieces, from a range of perspectives, on problematic, compulsive, disordered, risky, or excessive gambling. Jeffrey Christensen, Teresa McDowell, and Iva Kosutic provide a two-part comprehensive review of English-language scholarly discourses on psychological and relational approaches to the treatment of gambling disorder. Part 1 focuses on the "what" of knowledge production and treatment delivery by systematizing information on the types of scholarly articles that have been published in the last 50 years; the treatment approaches that have been researched and discussed; and the broader context of knowledge production, including in relation to the medicalization of mental health. Part 2 focuses on the "how" of treatment delivery, identifying a number of alternative treatment modalities in the last two decades including increased reliance on technology (i.e., internet and telephone/text) as an adjunct to face-toface treatment, or as a means for delivering stand-alone professionally facilitated or self-directed interventions. While discussing the benefits of these new approaches, the piece also situates their emergence within trends towards the growing use of technology, the prioritization of efficiency, and the on-going individual focus in mental health treatment provision. Rather than researching medical framings of problematic gambling, Annie-Claude Savard, Mélina Bouffard, Jean-Philippe Laforge and Sylvia Kairouz explore how a group of 30 young adult gamblers in Québec perceive the concept of responsibility. In this way they seek to augment, and critique, dominant understandings of responsible gambling by attending to the experiences of gamblers themselves. Fabio Lucchini and Simona Lorena Comi offer a further adaptation to contemporary work on gambling harm, through an estimate of the social costs of gambling harm in Italy. Using a comprehensive approach to cost modelling, incorporating estimated treatment costs for high-risk gamblers alongside costs of unemployment, family harm, and crime, this study suggests that the social costs of gambling in Italy exceed EUR 2.3 billion. Eva Samuelsson, Jukka Törrönen, Chiyoung Hwang, and Naoko Takiguchi offer an account of pachinko and pachislot in Japan gambling forms that are rarely considered in mainstream gambling literature, and that are often overlooked in gambling legislation and policy. Using group interviews with those who have experienced pachinko-related gambling problems, this study explores how people have dealt with shame, guilt, and stigma.

Moving away from a focus on at-risk gamblers, the final two research articles in our special issue address other, equally important dimensions of gambling studies. Pekka Sulkunen, Sebastien Berret, Virve Marionneau and Janne Nikkinen seek to understand better how gambling revenue is generated, and how it depends on product portfolios, operating costs, turnover, and the institutional contexts of the industry. Their article offers a comparative analysis of income statements from 30 European gambling companies, revealing intriguing patterns about how the surplus depends on volume, operating costs, monopoly status, and the game portfolio measured by aggregate returnto-players (RTP). Kathleen Maltzahn, John Cox, Sarah MacLean, Mary Whiteside and Helen Lee provide a narrative review of literature on bingo, a distinct, enduring, but (like, pachinko) understudied form of gambling that has been reshaped by technological and regulatory changes in many parts of the world. Besides offering crucial insights into bingo itself, the article suggests that close attention to bingo allows better understanding of groups of overlooked gamblers more 
generally (including Indigenous communities, and older women), and shows the value of methodological approaches to gambling studies that are congruent with the people and practices being studied.

We close the issue with a review essay by Fiona Nicoll, on State Looteries: Historical Continuity, Rearticulations of Racism and American Taxation (Kasey Henricks and David G. Embrick. Routledge, 2017). This important monograph uses the lens of critical race theory to provide an original, incisive account of how racial politics have driven and sustained lotteries in America since they were legalized in the 1960s. Nicoll's in-depth analysis of the book shows how it contributes a vital new lens on gambling and finance. Besides offering a robust account of how lotteries operate as a racialised tax transfer from black and brown citizens and communities, to the benefit of white citizens and communities, the books also provides concrete recommendations for lottery tax reform within a broader and diverse coalitional anti-racist politics.

Taken together, the articles and the review essay featured in this special issue range widely in geographical focus, in disciplinary grounding, and in approach to gambling and its related pleasures and harms. Along with our blog, and our special events, we hope that this eclectic and diverse mix of pieces gives a flavour of the sort of work we are seeking to support through our journal; we look forward to showcasing other examples in future issues.

\section{References}

Sarat, A., \& Silbey, S. (1988). The pull of the policy audience. Law \& Policy, 10 (2), 96-166. https://doi.org/10.1111/j.14679930.1988.tb00007.x

\section{Acknowledgements}

The authors would like to thank Elise Sammons and the members of the Editorial Board of Critical Gambling Studies for their comments on previous drafts of this statement, and their work to support the journal. 